\title{
Crecimiento de heteroestructuras de GaSb/GalnAsSb/GaSb por la técnica de Epitaxia en Fase Líquida
}

\section{Growth of GaSb/GalnAsSb/GaSb heteroestructure by Liquid Phase Epitaxy}

\author{
José Fernando Gómez*, Ana Patricia Cardona*, \\ Marianela de los Ríos**, Liliana Tirado Trujillo***, Hernando Ariza Calderón*****
}

Recibido: Mayo 6 de 2009

Aceptado: Octubre 22 de 2009

Correspondencia: Instituto Interdisciplinario de las Ciencias, Universidad del Quindio, Avenida Bolivar calle 12 norte Armenia Quindio. Email: labopt@uniquindio.edu.co

\section{RESUMEN}

En este trabajo se muestran algunos resultados preliminares del proceso de crecimiento por la técnica de Epitaxia en Fase Líquida (EFL) de la heteroestructura GaSb/GaInAsSb/GaSb. Se describe el proceso de fabricación de la heteroestructura así como también se muestran los resultado obtenidos de su caracterización por Difracción de Rayos X (DRX) y Fotoluminiscencia (FL) los cuales evidencian el crecimiento epitaxial de la heteroestructura.

Palabras clave: Semiconductores, heteroestructura, EFL, DRX, FL.

\section{ABSTRACT}

In this paper we show some preliminary results of the liquid phase epitaxy growth process for the heterostructures GaSb/GaInAsSb/GaSb. We describe the fabrication process of the heterostructures and also we present the analysis of its X-Ray Diffraction patterns (XRD) and Photoluminescence (PL) characterization. These results evidenced the epitaxial growth of the heterostructures.

Key words: Semiconductors, heteroestructure, $L P E, X R D, P L$.

\section{INTRODUCCIÓN}

$\mathrm{E}$ I descubrimiento y posterior desarrollo de los materiales semiconductores ha revolucionado la historia de la humanidad, ya que permitió la miniaturización y desarrollo de los más diversos dispositivos electrónicos, situación que promovió de manera vertiginosa el proceso de globalización.

Unas de las configuraciones que han impactado de manera favorable el desarrollo tecnológico en las últimas décadas han sido las heteroestructuras, las cuales ofrecen una amplia aplicabilidad y permiten obtener dispositivos digitales y analógicos para trabajar en altas frecuencias. Estas consisten básicamente en la unión de dos o más semiconductores con diferente composición química. Las propiedades de estas heteroestructuras dependen de múltiples factores como el espesor de las capas fabricadas, el diseño de las capas, el método de fabricación y especialmente los materiales utilizados.
Por sus potenciales aplicaciones optoelectrónicas [1,2] como son las celdas termofotovoltaicas[3,4], fotodiodos, láseres semiconductores $[5,6]$ y detectores, las heteroestructuras basadas en GaSb han sido objeto de estudio durante las últimas decadas y se han propuesto nuevas configuraciones con el fin de lograr una mejor eficiencia en los dispositivos basados en este material. Particularmente la heteroestructura de GaSb/GalnAsSb/GaSb (Figura 1) sucitado un gran interés debido a que ha permitido mejorar notablemente la eficiencia cuántica de las celdas termofotovoltaicas $[7,8,9,10]$.

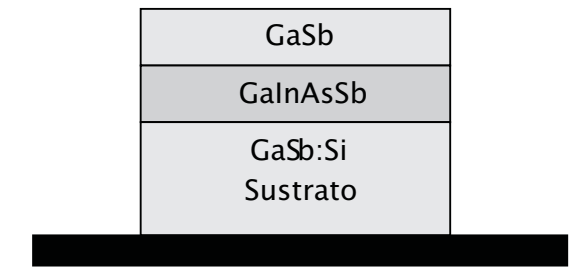

Figura 1. Esquema de la heteroestructura $\mathrm{GaSb} / \mathrm{GaInAsSb/GaSb}$.

\footnotetext{
* Estudiante de Maestría en Ciencia de los Materiales, Universidad del Quindío.

** Magister en Ciencias de los Materiales, Universidad del Quindío.

*** Docente Investigadora, Universidad del Quindío.

**** Director Instituto Interdisciplinario de las Ciencias, Universidad del Quindío.
} 
Una de las técnicas implementadas para la fabricación de materiales semiconductores III-V, es la Epitaxia en Fase Líquida (EFL), la cual consiste en el crecimiento de una película epitaxial sobre un sustrato monocristalino a partir de una solución líquida, saturada o súper-saturada con el material a crecer. El sustrato debe tener una estructura cristalina y parámetro de red similares a los de la película, de manera que se permita una continuación coherente de la estructura cristalina del sustrato en la película, pues de lo contrario, no se dará un crecimiento epitaxial. El término epitaxia (del griego epi: sobre, taxis: orden) fue presentado por primera vez en 1928 por Royer [11]. Este término se refiere a un proceso de crecimiento orientado cristalográficamente de una película sobre un sustrato. A estos procesos se les llama, en forma genérica, métodos de crecimiento epitaxial de películas o métodos de crecimiento de películas epitaxiales. Las capas formadas por técnicas epitaxiales se conocen como heteroepitaxiales cuando el sustrato es de un material diferente al de la película que crece sobre él, mientras que las homoepitaxiales están formadas por películas del mismo material que el del sustrato.

En este trabajo presentamos los avances obtenidos en el proceso de fabricación de la heteroestructura GaSb/GalnAsSb/GaSb por la técnica de EFL con la cual se cuenta en el Laboratorio de Optoelectrónica del Instituto Interdisciplinario de las Ciencias de la Universidad del Quindío.

\section{METODOLOGÍA}

Para el crecimiento de las heteroestructuras se utilizaron sustratos monocristalinos comerciales de GaSb:Si con orientación cristalográfica (100). Previo al crecimiento, el sustrato fue sometido a un tratamiento químico de desengrase con acetona caliente, metanol caliente e isopropanol a temperatura ambiente, posteriormente se realizó un proceso de decapado con una mezcla de ácido tartárico, fluorhídrico, peróxido de hidrógeno y agua desionizada, con el objetivo de remover los óxidos nativos del material.

La heteroestructura de GaSb/GalnAsSb/GaSb fue crecida utilizando un sistema de EFL convencional, con bote de grafito horizontal y regleta deslizante (Figura 2.), en atmósfera de hidrógeno purificado. La solución cuaternaria utilizada es una solución rica en indio en la cual se emplearon materiales con una pureza de $6 \mathrm{~N}$ para el $\mathrm{Sb}, 5 \mathrm{~N}$ para el $\mathrm{In}, 7 \mathrm{~N}$ para el Ga y GaAs sin dopar. Los materiales fueron pesados en una balanza de alta precisión $(0.01 \mathrm{mg}$ ) y cargados en el bote de grafito para ser sometidos a un recocido a $700^{\circ} \mathrm{C}$ durante 18 horas con el fin de reducir los óxidos presentes y homogenizar la solución.
Paralelo a la preparación de la solución cuaternaria se realiza la preparación de la solución binaria utilizada en el crecimiento de la capa final de la heteroestructura. Esta es una solución rica en galio y los elementos utilizados tienen las mismas características de pureza que los empleados en la solución cuaternaria. Esta solución es sometida al mismo tratamiento térmico de la solución cuaternaria. Las soluciones (binaria y cuaternaria) se encuentran cada una en cavidades diferentes del bote de grafito, separadas entre sí por una cavidad desocupada.

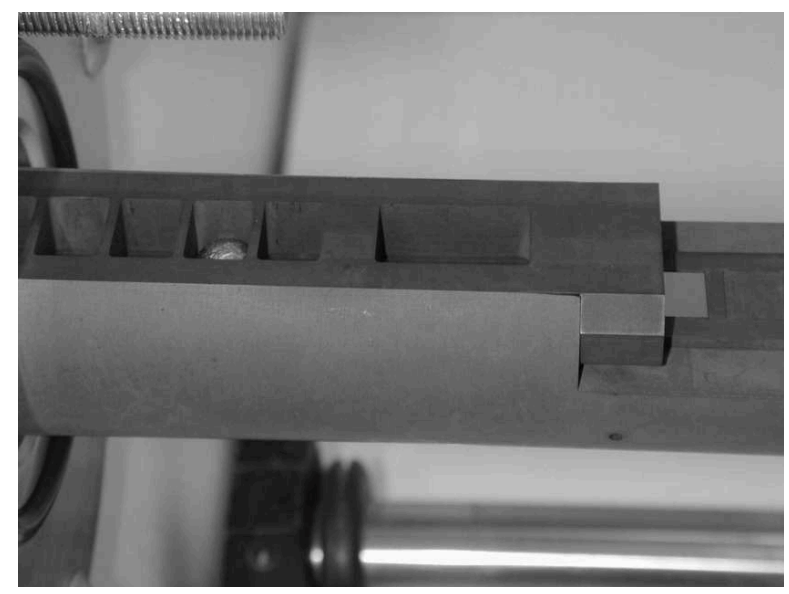

Figura 2. Bote de grafito con regleta deslizante, conteniendo solución en la cavidad No. 2 y sustrato sobre la regleta.

Después de preparar las soluciones y cargar el sustrato en la regleta deslizante, este último es sometido a un tratamiento térmico a $640^{\circ} \mathrm{C}$ en atmósfera de hidrógeno durante una hora. Luego se corre un programa de temperatura donde ésta disminuye a una razón de $2^{\circ}$ por minuto hasta alcanzar una temperatura de estabilización de $540^{\circ} \mathrm{C}$, la cual es mantenida por 2 horas. Una vez transcurrido este tiempo se inicia una nueva rampa de enfriamiento de $0.3^{\circ} \mathrm{C} / \mathrm{min}$. Cuando se llega a la temperatura de $529.8^{\circ} \mathrm{C}$ se desliza el sustrato debajo de la primera solución (cuaternaria) para dar inicio al crecimiento de la primera capa; el tiempo de contacto es de dos minutos. Pasado el tiempo de contacto con la primera solución el sustrato se desliza debajo de la cavidad desocupada que separa las dos soluciones, mientras que la temperatura sigue disminuyendo con la misma razón de cambio. Cuando se llega a la temperatura de $525^{\circ} \mathrm{C}$ se desliza nuevamente el sustrato hacia la solución binaria, para dar inicio al crecimiento de la última capa de la heteroestructura. En la Figura 3 se muestra un diagrama de flujo generalizado para el crecimiento de películas por la técnica Epitaxia en Fase Líquida.

La heteroestructura fabricada fue caracterizada estructuralmente por Difracción de Rayos $X$ 
en un difractómetro Bruker D8 Advance con cátodo de Cu y detector de centelleo, utilizando la configuración $\theta-2 \theta$ realizando un barrido de $20^{\circ}$ a $80^{\circ}$ con un paso de 0.01 grados y un tiempo por paso de 2 segundos.

La caracterización óptica se realizó por medio de la técnica de fotoluminiscencia, utilizado un láser de Argón en la línea 488, el cual permite variar la potencia de salida entre 2 y $40 \mathrm{~mW}$. La muestra fue ubicada en un portamuestras de cobre en el extremo de un dedo frío conectado a un crióstato con sistema cerrado de $\mathrm{He}$, el cual permite obtener una temperatura estable de 10K. El pre vacío del sistema se realizó con una bomba mecánica Franklin Electric, para obtener una presión de $2 \times 10^{-3}$ Torr. Un controlador de temperatura LakeShore 330, controla dos sensores de Si y permite obtener temperaturas entre 11 y $300 \mathrm{~K}$, con una precisión de 0,2K. La señal fotoluminiscente de la muestra se lleva a un espectrómetro Spex $500 \mathrm{~m}$ con una resolución de $0.2 \mathrm{~A}^{\circ}$, el cual contiene una rejilla de difracción de 600 $\mathrm{ran} / \mathrm{mm}$.

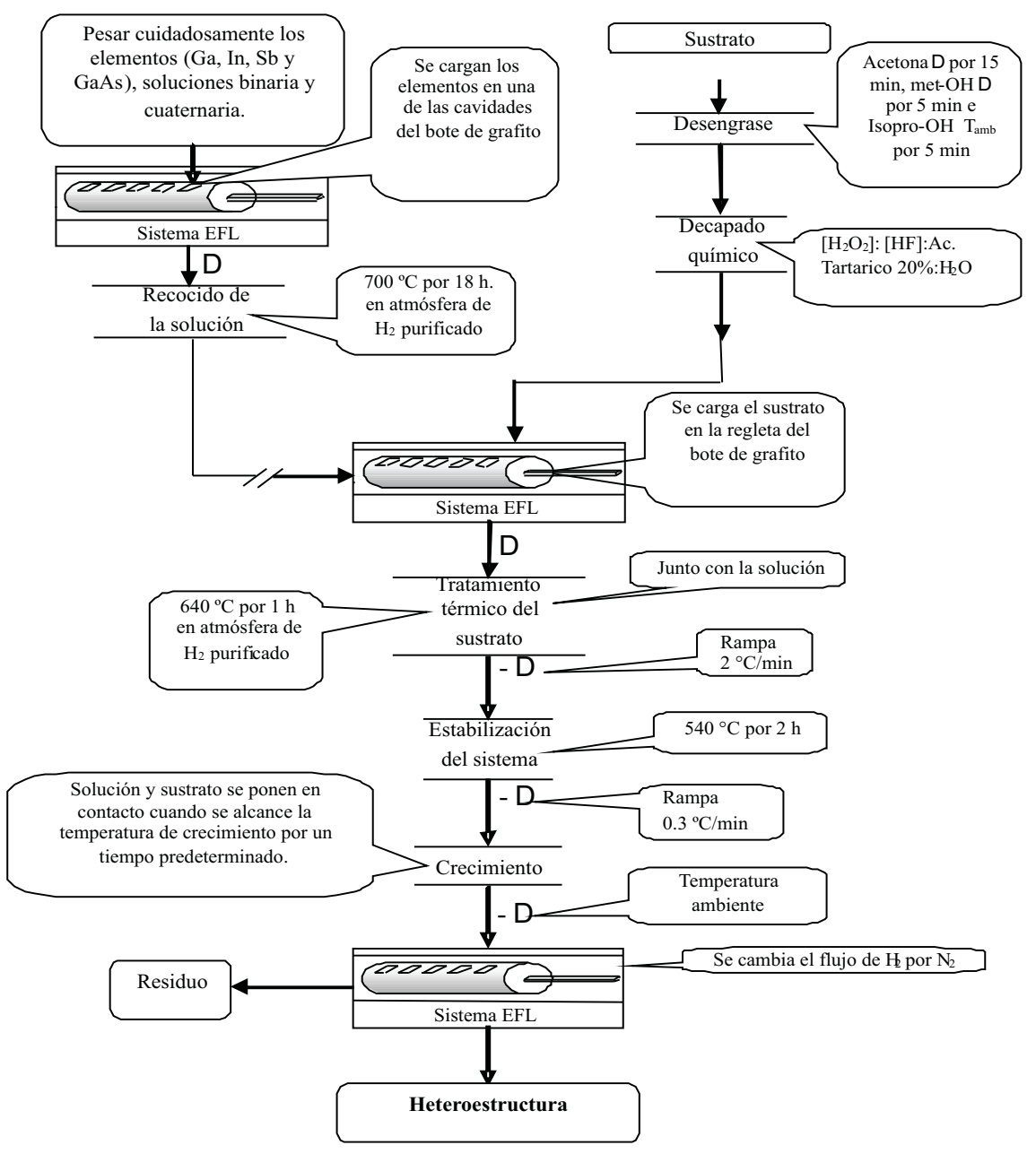

Figura 3. Diagrama de flujo para el crecimiento de películas epitaxiales por la técnica de EFL.

Para la detección de la radiación proveniente de la muestra se utilizó un detector Hamamatsu de GalnAs. Este detector está acoplado a un amplificador Lock-in, que sintonizado a $83 \mathrm{~Hz}$ con el chopper, permite eliminar el ruido proveniente de fuentes externas. Todos los parámetros se dejaron constantes para efectos comparativos en cada uno de los espectros, haciendo variaciones únicamente en potencia. Finalmente, el computador hace un barrido automático de longitudes de onda con sus correspondientes intensidades para cada rango espectral.

\section{RESULTADOS Y DISCUSIÓN}

En la Figura 4 se observa una imagen de una de las muestras fabricadas. En su superficie se pueden apreciar algunas irregularidades causadas por el arrastre de solución. 


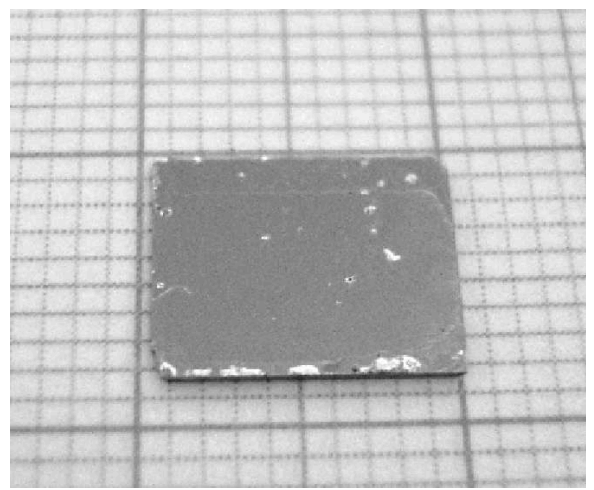

Figura 4. Fotografía de una heteroestructura fabricada por la técnica de EFL.

En la Figura 5 se presenta una comparación entre los difractogramas del sustrato y de la heteroestructura fabricada. En estos se observan los máximos de difracción del GaSb con la orientación preferencial (100) como era de esperarse de acuerdo con el sustrato utilizado en el crecimiento. Estos resultados se confirmaron con la base de datos del equipo, coincidiendo con el PDF 07-0215 (Powder Diffraction File Database) para el GaSb.

La asimetría presente en los picos (200) y (400) del difractograma de la heteroestructura se puede atribuir a pequeñas diferencias en el parámetro de red del sustrato y de las capas, esto evidencia un crecimiento epitaxial de las películas cuaternaria y binaria así como una buena calidad cristalina de éstas, corroborándose con los resultados obtenidos por fotoluminiscencia.

Los picos (111) y (222) son atribuidos a una orientación preferencial de los cúmulos formados por el arrastre de solución en la superficie de la muestra.

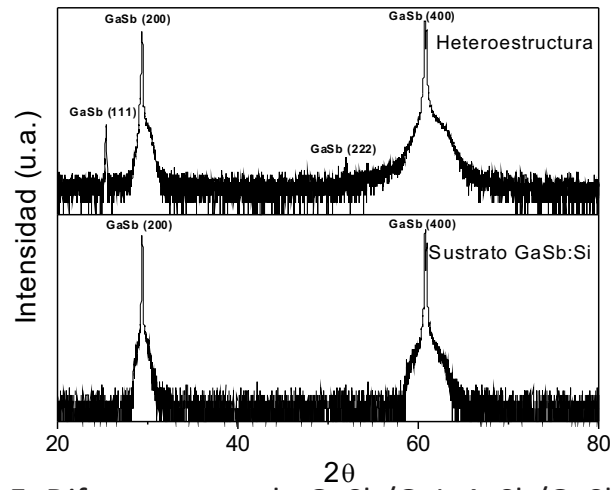

Figura 5. Difractograma de GaSb/GalnAsSb/GaSb y del sustrato $\mathrm{GaSb}: \mathrm{Si}$

Con el fin de complementar el estudio de las heteroestructuras, se realizó un análisis por medio de Fotoluminiscencia, donde se pudo obtener información acerca de la calidad cristalina y los picos característicos de la muestra. La figura 6 presenta el espectro de fotoluminiscencia de la heteroestructura GaSb/GalnAsSb/GaSb:Si a una temperatura de 11K, con una potencia de excitación de $40 \mathrm{~mW}$. En esta figura, se han incluido los espectros de GaSb sin dopar y el de GalnAsSb/GaSb:Si con el fin de comparar su respuesta fotoluminiscente.

El espectro de la heteroestructura presenta tres bandas principales, de las cuales la de menor energía, más intensa, está relacionada con el cuaternario GalnAsSb. En color negro se ha montado el espectro del GalnAsSb crecido sobre el sustrato de $\mathrm{GaSb}: \mathrm{Si}$. En este espectro se puede observar un corrimiento del pico principal, asociado con una mayor intensidad del pico excitónico en la heteroestructura. En el caso de cuaternario la transición más intensa corresponde a una transición que involucra estados ligados.

Las bandas de mayor energía, asociadas con el binario GaSb está compuesta por dos transiciones principales, asociadas a excitones ligados y transición donador-aceptor [12]. Al comparar el espectro de la heteroestructura con el del binario, se puede observar que las transiciones excitónicas son más intensas que las donador-aceptor, reflejando el mejoramiento de la película al crecer la heteroestructura.

El ancho del pico excitónico refleja la calidad cristalina de la muestra, pues a medida que se perturba el excitón con efectos estadísticos de tensiones, campos eléctricos, desorden en la red, dislocaciones e imperfecciones, más anchos serán esos picos por las variaciones energéticas que originan esas perturbaciones en algunos excitones antes de recombinarse. Los anchos obtenidos en los espectros de la Figura 6 (alrededor de $11 \mathrm{meV}$ ), sumados a la buena definición de las transiciones luminiscentes son indicio de la buena calidad cristalina de las películas crecidas en el sistema de EFL del Instituto Interdisciplinario de las Ciencias.

La variación de los espectros fotoluminiscentes con la potencia nos permite obtener mayor información acerca de las transiciones involucradas en los espectros. En la Figura 7 se muestra el espectro fotoluminiscente de la heteroestructura al variar la potencia. En el recuadro se ha incluido la emisión fotoluminiscente del pico más intenso en función de la potencia de excitación. El resultado obtenido de esta gráfica nos permitió obtener información acerca de los estados involucrados en la respuesta espectral. El valor de la pendiente obtenida del ajuste lineal da un valor igual a 1.9, asociado atransiciones cuyos estados involucrados son libres o estados ligados a las bandas, correspondiéndose con los resultados obtenidos anteriormente, donde la superficie mejora al crecer la heteroestructura. 


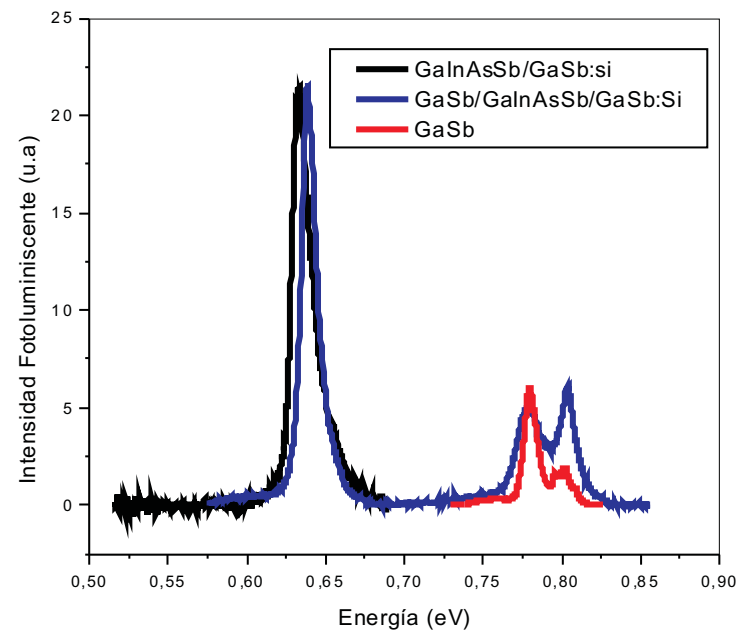

Figura 6. Espectros de FL para GaSb/GalnAsSb/GaSb, GalnAsSb/GaSb:Si y el sustrato sobre el cual se creció la heteroestructura, utilizando una potencia de $40 \mathrm{~mW}$ y a $11 \mathrm{~K}$.

\section{CONCLUSIONES}

De acuerdo a los resultados obtenidos por difracción de rayos $X$ y fotoluminiscencia, las películas que forman la heteroestructura presentan una alta calidad cristalina, lo que permite concluir que se encontraron los parámetros adecuados para fabricar el sistema GaSb/GalnAsSb/GaSb.

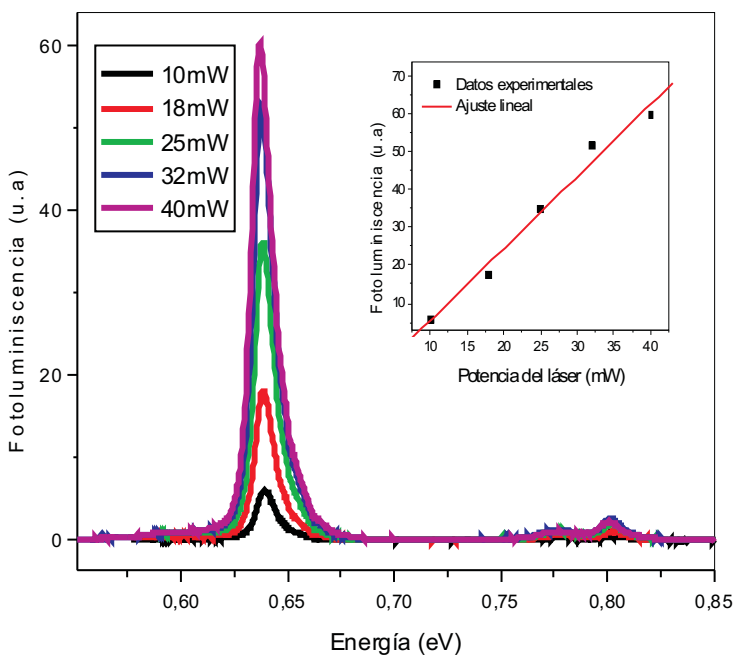

Figura 7. Espectros de FL para GaSb/GalnAsSb/GaSb:Si al variar la potencia. En el recuadro se muestra la intensidad del pico de GalnAsSb en función de la potencia.

Del análisis de los espectros de fotoluminiscencia se observó que el GalnAsSb emite con mayor intensidad cuando está recubierto por una capa de GaSb. Es decir, en la heteroestructura se obtiene una mayor emisión que en el sistema GalnAsSb/GaSb:Si significando esto que la superficie del cuaternario se pasiva al crecer sobre ella una película de GaSb. Este recubrimiento no sólo evita la oxidación del GalnAsSb si no también, disminuye los defectos de superficie.

\section{BIBLIOGRAFÍA}

(1) Manz C, Yang Q, Rattunde M, Schulz N, Rösener B, Kirste L, et al. J. Crys. Growth 2009; 311:1920.

(2) Manz C, Köhler K, Kirste L, Yang Q, Rösener B, Moser B et al. J. Crys. Growth 2009; 311:4158.

(3) Qui K, S. Hayden AC, Mauk MG, Sulima OV. Solar Energy Materials and Solar Cells 2006; 90:68.

(4) Charache GW, Baldasaro PF, Danielson LR, DePov DM, Freeman MJ, Wang CA et al., J. Appl. Phys 1999; 85:2247.

(5) Ahmetoglu M, Kaynak G, Andreev IA, Kunitsyna EV, Mikhailova MP and Yakovlev YP. Technical Physics Letters 2008; $34: 937$.

(6) Cerutti, G. Boissier, P. Grech, A. Perona, J. Angellier, Y. Rouillard, al. J. Crys. Growth 2007; 301:967.

(7) Lei L, Nuofu C, Xiaoli Y, Yu W, and Fubao G. J. of Semiconductors 2008; 29:1258.

(8) Ahmetoglu M. Thin Solid Films 2008; 516:1227.

(9) Wang CA, Shiau DA, Calawa DR. J. Crys. Growth 2004; 261:372.

(10) Kumara A and Dutta PS. J. Crys. Growth 2008; 310: 1647.

(11) Royer L. Bull. Soc. Fr. Miner. 1928; 51:7.

(12) Dutta P. S. and Bhat H.L.,J. Appl. Phys 81 (1997) 5821. 